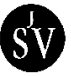

\title{
DYNAMIC RESPONSE OF A HORIZONTALLY CURVED BEAM SUBJECTED TO VERTICAL AND HORIZONTAL MOVING LOADS
}

\author{
Y.-B. YANG AND C.-M. WU \\ Department of Civil Engineering, National Taiwan University, Taipei, Taiwan 10617, Republic of China, \\ E-mail: ybyang@ce.ntu.edu.tw \\ AND \\ J.-D. YAU
}

Department of Architecture and Building Technology, Tamkang University, Taipei, Taiwan 106, Republic of China

(Received 16 November 1999, and in final form 10 October 2000)

\begin{abstract}
Analytic solutions are derived for a horizontal curved beam subjected to vertical and horizontal moving loads. The horizontal moving loads may be regarded as the centrifugal forces generated by vehicles moving along a curved path, which were rarely studied by previous researchers. Both a single moving load and a series of equidistant moving loads are considered. By superposition of the waves generated by consecutively moving loads on the curved beam, the conditions for the resonance and cancellation phenomena to occur are established. Compared with the numerical approaches, the present approach has the advantage that it provides clear physical insights into the various vehicle-induced phenomena on curved beams, while allowing us to identify the key parameters involved.

(C) 2001 Academic Press
\end{abstract}

\section{INTRODUCTION}

The vehicle-induced vibration on bridges has been a subject of interest for more than one and half centuries. The pioneering works on this subject include those of Willis [1] and Stokes [2] following the collapse of the Chaster Rail Bridge in England in 1847. The problem of simple beams under moving vehicular loads was studied in 1922 by Timoshenko neglecting the inertia effect of the vehicle [3]. In addition to the inertia of the beam, the inertia of the vehicle was included by Jeffcott in his study in 1929 [4], followed by the works of Stanišić and Hardin [5] and Ting et al. [6]. By taking into account the suspension properties of the vehicle, the sprung mass model was adopted in the studies by Tan and Shore [7], Veletsos and Huang [8], Blejwas et al. [9], etc. An early treatise on this subject that covers vehicle models of various complexities is the book by Frýba in 1972 [10]. In the past two decades, research on the vehicle-induced vibrations of bridges continued to grow, which was generally directed toward the use of more realistic vehicle modes $[11,12]$ and bridge models $[13,14]$, the inclusion of surface roughness $[12,15]$, or the implementation of efficient solution schemes including the use of vehicle-bridge interaction (VBI) elements $[16,17]$. Some partial reviews of the research on vehicle-induced vibrations of the bridge are 
available in references [18-20]. In a recent book by Frýba on the dynamics of railway bridges, 231 references have been cited [21].

Previously, a great portion of the research on vehicle-induced vibrations has been conducted for straight beams. While some research has been conducted for horizontally curved beams under moving loads [7, 22, 23], concern was generally placed on the vertical or out-of-plane vibration of the curved beam. To the knowledge of the writers, the radial or in-plane vibration of curved beams under the action of centrifugal forces, which are generated by masses moving along circular paths, has rarely been studied. Just as a vertical moving load will cause some impact on the vertical oscillation of a straight beam, so too, a centrifugal force generated by a mass moving over a horizontally curved beam will induce certain impact on the radial response of the beam. The objective of this paper is to establish a complete theory for treating the vibration of horizontally curved beams subjected to the moving masses, each of which will be simulated as a pair of gravitational force and centrifugal force. The conditions for superposition of the waves generated by consecutively moving loads to result in the phenomena of resonance and cancellation on the curved beam will be identified. The capability and reliability of the present theory will be demonstrated in the exemplary studies.

\section{GOVERNING DIFFERENTIAL EQUATIONS}

Consider the horizontally curved beam in Figure 1, in which $\varphi$ denotes the subtended angle, $R$ the radius of curvature, and $L$ the length of the beam. A right-handed coordinate system is chosen, the $y$ - and $z$-axis of which coincide with the principal axis of the cross-section, and the $x$-axis is tangent to the centroidal axis of the beam. Let $u_{x}, u_{y}$ and $u_{z}$ denote the displacements of the centroid of each cross-section of the curved beam along the three axes, and $\theta_{x}, \theta_{y}$ and $\theta_{z}$ the rotations about the three axes. All the deformations are assumed to be small so that the linear theory applies. The curved beam is assumed to be made of constant, bisymmetric cross-sections with negligible warping resistance. The following are the linear differential equations for the curved beam [24, 25].

$$
\begin{aligned}
& \text { Axial displacement: } \quad E A\left(u_{x}^{\prime \prime}+\frac{u_{z}^{\prime}}{R}\right)=0 \\
& \text { Radial displacement: } E I_{y}\left(u_{z}^{\prime \prime \prime \prime}+2 \frac{u_{z}^{\prime \prime}}{R^{2}}+\frac{u_{z}}{R^{4}}\right)+\frac{E A}{R}\left(u_{x}^{\prime}+\frac{u_{z}}{R}\right)=0 \\
& \text { Vertical displacement: } \quad E I_{z}\left(u_{y}^{\prime \prime \prime \prime}-\frac{\theta_{x}^{\prime \prime}}{R}\right)-\frac{G J}{R}\left(\theta_{x}^{\prime \prime}+\frac{u_{y}^{\prime \prime}}{R}\right)=0 \\
& \text { Torsional rotation: } \frac{E I_{z}}{R}\left(u_{y}^{\prime \prime}-\frac{\theta_{x}}{R}\right)+G J\left(\theta_{x}^{\prime \prime}+\frac{u_{y}^{\prime \prime}}{R}\right)=0
\end{aligned}
$$

where a prime denotes differentiation with respect to the axis $x, E$ and $G$ denote the moduli of elasticity and rigidity, respectively, of the beam, $A$ the cross-sectional area, $I_{y}$ and $I_{z}$, respectively, the moments of inertia about the $y$ - and $z$-axis, and $J$ the torsional constant. From equations (1)-(4), one can observe that the differential equations for the in-plane displacements, i.e., $u_{x}$ and $u_{z}$, are independent of those for the out-of-plane displacement, i.e., $u_{y}$ and $\theta_{x}$. Furthermore, the differential equation for the axial displacement $u_{x}$ and that for the radial displacement $u_{z}$ are coupled, and the same is true for the vertical displacement $u_{y}$ and torsional rotation $\theta_{x}$. 


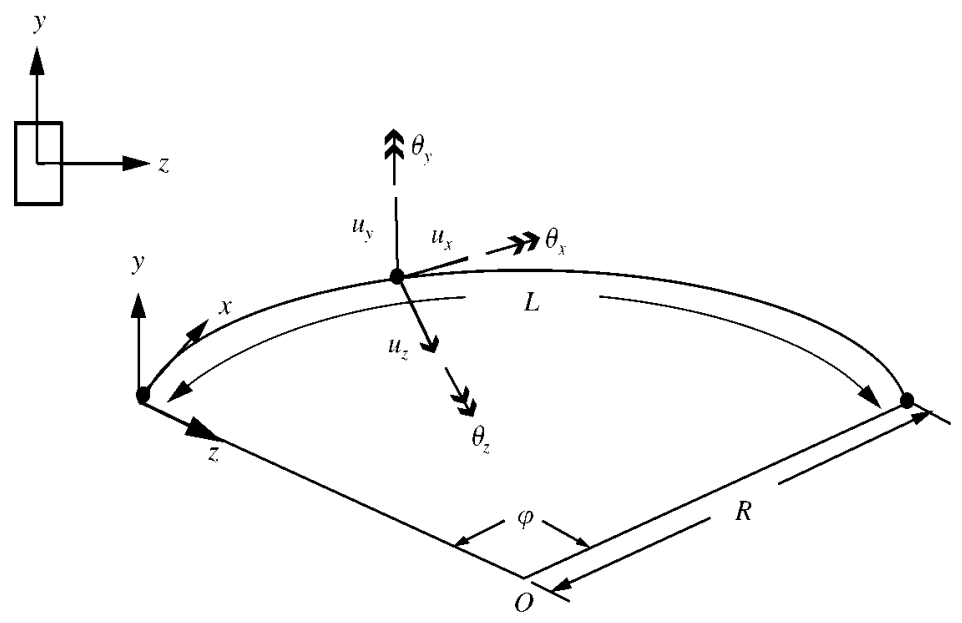

Figure 1. Co-ordinates of curved beam.

\section{CURVED BEAM SUBJECTED TO A SINGLE MOVING LOAD}

The two ends of the curved beam are assumed to be simply supported, in the sense that the flexural displacements and twisting rotation of the beam are restrained at the supports, but their first derivatives are not zero. In general, the action of the moving vehicle can be replaced by a vertical moving load, to simulate the gravitational effect, and a horizontal moving load, to simulate the centrifugal effect, as shown in Figure 2(a).

\subsection{CURVED BEAM SUBJECTED TO A VERTICAL MOVING LOAD}

As the in-plane and out-of-plane behaviors of the curved beam are uncoupled, we shall consider first the vertical vibration of the curved beam under the action of a vertical moving load (Figure 2(c)). Let $m_{v}$ denote the mass of the vehicle moving at speed $v$. The load of the vehicle is $f_{v}=-m_{v} g$, where $g$ is the acceleration due to gravity. By taking into account the effect of inertia, the equations of motion for the vertical vibration of the curved beam can be modified from equations (3) and (4) as follows:

$$
\begin{aligned}
& m \ddot{u}_{y}+E I_{z}\left(u_{y}^{\prime \prime \prime \prime}-\frac{\theta_{x}^{\prime \prime}}{R}\right)-\frac{G J}{R}\left(\theta_{x}^{\prime \prime}+\frac{u_{y}^{\prime \prime}}{R}\right)=f_{v} \delta(x-v t), \\
& \rho J \ddot{\theta}_{x}+\frac{E I_{z}}{R}\left(u_{y}^{\prime \prime}-\frac{\theta_{x}}{R}\right)+G J\left(\theta_{x}^{\prime \prime \prime}+\frac{u_{y}^{\prime \prime}}{R}\right)=0,
\end{aligned}
$$

where $m$ denotes the mass per unit length, $\rho$ the density of the curved beam and $\delta$ is Dirac's delta function. The term on the right-hand side of equation (5a) represents the effect of the vertical moving load $f_{v}$, where $f_{v}=-m_{v} g$. For the present problem, the vertical displacement $u_{y}$ can be expressed as the summation of a series of sine functions that satisfy the boundary conditions

$$
u_{y}(x, t)=\sum_{i=1}^{\infty} q_{y i}(t) \sin \frac{\mathrm{i} \pi x}{L}
$$




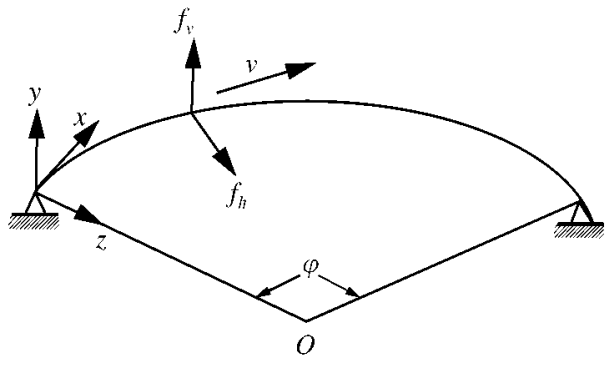

(a)

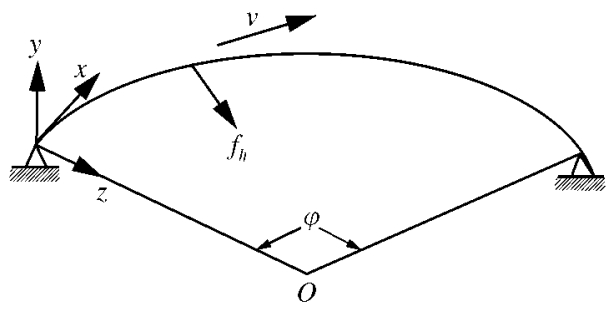

(b)

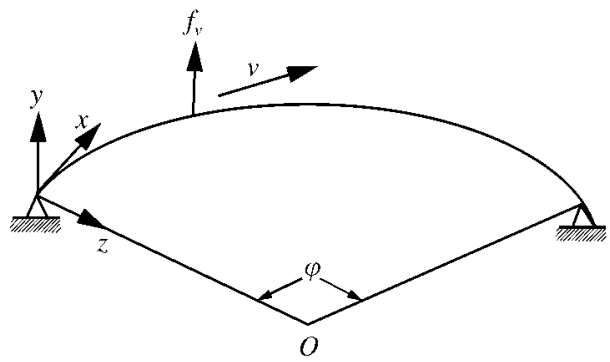

(c)

Figure 2. Curved beam subjected to moving loads: (a) general; (b) horizontal; and (c) vertical.

where $q_{y i}$ denotes the generalized co-ordinate for the $i$ th mode. The expression for the angle of twist $\theta_{x}$ is not arbitrary, but must be determined from equation (4). Substituting equation (6) into equation (4) and making use of boundary conditions for twisting, one obtains

$$
\theta_{x}(x, t)=\sum_{i=1}^{\infty} \gamma_{i} q_{y i}(t) \sin \frac{\mathrm{i} \pi x}{L}
$$

where $\gamma_{i}=-R(i \pi / L)^{2}\left(G J+E I_{z}\right) /\left[(\mathrm{i} \pi / L)^{2} G J R^{2}+E I_{z}\right]$, or equivalently,

$$
\theta_{x}(x, t)=\sum_{i=1}^{\infty} q_{\theta i}(t) \sin \frac{\mathrm{i} \pi x}{L},
$$

where $q_{\theta i}$ denotes the $i$ th generalized coordinate for the angle of twist $\theta_{x}$. For the present purposes, let us consider only the contribution of the first modes, i.e.,

$$
u_{y}(x, t)=q_{y 1}(t) \sin \frac{\pi x}{L}, \quad \theta_{x}(x, t)=q_{\theta 1}(t) \sin \frac{\pi x}{L},
$$

where $q_{y 1}$ and $q_{\theta 1}$ denote the first generalized co-ordinates for $u_{y}$ and $\theta_{x}$, respectively.

To solve the two differential equations in (5), Galerkin's method will be used. First, one multiplies both sides of equation (5a) by the variation $\delta u_{y}$ and those of equation (5b) by $\delta \theta_{x}$. Use the first-mode approximations for $u_{y}$ and $\theta_{x}$ in equations (9). Then integrate the two differential equations each with respect to $x$ from 0 to $L$. The result is

$$
\begin{aligned}
& \left(\ddot{q}_{y 1}+a_{1} q_{y 1}+a_{2} q_{\theta 1}\right) \delta q_{y 1}=\frac{2 f_{y}}{m L} \sin \frac{\pi v t}{L} \delta q_{y 1}, \\
& \left(\ddot{q}_{\theta 1}+b_{1} q_{\theta 1}+b_{2} q_{y 1}\right) \delta q_{\theta 1}=0,
\end{aligned}
$$


where

$$
\begin{aligned}
& a_{1}=-\frac{1}{\rho J}\left[\frac{E I_{z}}{R^{2}}+G J\left(\frac{\pi}{L}\right)^{2}\right], \quad a_{2}=-\frac{1}{\rho J} \frac{1}{R}\left(\frac{\pi}{L}\right)^{2}\left(E I_{z}+G J\right), \\
& b_{1} \sim \frac{1}{m}\left(\frac{\pi}{L}\right)^{2}\left[E I_{z}\left(\frac{\pi}{L}\right)^{2}+\frac{G J}{R^{2}}\right], \quad b_{2}=\frac{1}{m R}\left(\frac{\pi}{L}\right)^{2}\left(E I_{z}+G J\right) .
\end{aligned}
$$

Since the variations $\delta q_{y 1}$ and $\delta q_{\theta 1}$ are arbitrary, equations (10) reduce to

$$
\ddot{q}_{y 1}+a_{1} q_{y 1}+a_{2} q_{\theta 1}=\frac{2 f_{v}}{m L} \sin \frac{\pi v t}{L}, \quad \ddot{q}_{\theta 1}+b_{1} q_{\theta 1}+b_{2} q_{y 1}=0 .
$$

The general solutions to equations (12) are composed of two parts, i.e., the homogenous solutions and the particular solutions,

$$
q_{y 1}=q_{y 1 h}+q_{y 1 p}, \quad q_{\theta 1}=q_{\theta 1 h}+q_{\theta 1 p},
$$

where the subscripts $h$ and $p$, respectively, denote the homogeneous and particular solutions.

The homogeneous solutions can be given as follows:

$$
q_{y 1 h}=h_{1} \sin \omega_{v 1} t+h_{2} \cos \omega_{v 1} t, \quad q_{\theta 1 h}=k_{1} \sin \omega_{v 1} t+k_{2} \cos \omega_{v 1} t,
$$

where $\omega_{v 1}$ denotes the fundamental frequency of vibration for the vertical direction of the curved beam and $h_{1}, h_{2}, k_{1}, k_{2}$ are the coefficients to be determined from the initial conditions. By substituting equations (14) into equations (12) and dropping the term containing $f_{v}$, one obtains

$$
\left[\begin{array}{cc}
a_{1}-\omega_{v 1}^{2} & a_{2} \\
b_{2} & b_{1}-\omega_{v 1}^{2}
\end{array}\right]\left\{\begin{array}{l}
q_{y 1 h} \\
q_{\theta 1 h}
\end{array}\right\}=\left\{\begin{array}{l}
0 \\
0
\end{array}\right\} .
$$

By letting the determinant in equation (15) equal zero, the fundamental frequency $\omega_{v 1}$ can be solved as [26]

$$
\omega_{v 1}=\sqrt{\frac{a_{1}+b_{1}+\sqrt{\left(a_{1}-b_{1}\right)^{2}+4 a_{2} b_{2}}}{2}} .
$$

As for the particular solutions, the following may be assumed:

$$
q_{y 1 p}=p_{y 1} \sin \frac{\pi v t}{L}, \quad q_{\theta 1 p}=p_{\theta 1} \sin \frac{\pi v t}{L}
$$

where $p_{y 1}$ and $p_{\theta 1}$ denote the amplitudes of vibration. Substituting equations (17) into equations (10) yields

$$
\left[\begin{array}{cc}
a_{1}-(\pi v / L)^{2} & a_{2} \\
b_{2} & b_{1}-(\pi v / L)^{2}
\end{array}\right]\left\{\begin{array}{c}
p_{y 1} \\
p_{\theta 1}
\end{array}\right\}=\left\{\begin{array}{c}
\frac{2 f_{v}}{m L} \\
0
\end{array}\right\}
$$


From the preceding equation, $p_{y 1}$ can be solved:

$$
p_{y 1}=\frac{2 f_{v}}{m L} \frac{1}{\omega_{v 1}^{2}} \frac{1}{1-S_{v 1}^{2}} \beta,
$$

where

$$
S_{v 1}=\frac{\pi v}{L \omega_{v 1}}, \quad \beta=\frac{b_{1}-(\pi v / L)^{2}}{b_{1}+a_{1}-\omega_{v 1}^{2}-(\pi v / L)^{2}} .
$$

Here, $S_{v 1}$ denotes the speed parameter for the vertical vibration of the curved beam, which represents the ratio of the driving frequency, $\pi v / L$, implied by the moving load to the fundamental frequency of the beam, $\omega_{v 1}$.

The initial displacement and velocity of the beam are zero before it is subjected to the moving load. Such conditions should be obeyed by the general solution $q_{y 1}$ given in equation (13), or the sum of $q_{y 1 h}$ and $q_{y 1 p}$ given in equations (14a) and (17a), respectively. By these conditions, the coefficients of the homogeneous solution can be determined as $h_{1}=-p_{y 1} S_{v 1}$ and $h_{2}=0$. It follows that the general solution $q_{y 1}$ is

$$
q_{y 1}(t)=p_{y 1}\left(\sin \frac{\pi v t}{L}-S_{v 1} \sin \omega_{v 1} t\right) .
$$

By the relation $f_{v}=-m_{v} g$ and equation (19), the preceding equation may be rewritten as

$$
q_{y 1}(t)=-\frac{2 m_{v} g}{m L} \frac{1}{\omega_{v 1}^{2}} \frac{1}{1-S_{v 1}^{2}} \beta \Psi_{v 1}(t),
$$

where the amplitude function $\Psi_{v 1}(t)$ is

$$
\Psi_{v 1}(t)=\sin \frac{\pi v t}{L}-S_{v 1} \sin \omega_{v 1} t .
$$

Consequently, the vertical displacement of the curved beam is

$$
u_{y}(x, t)=-\frac{2 m_{v} g}{m L} \frac{1}{\omega_{v 1}^{2}} \frac{1}{1-S_{v 1}^{2}} \beta \Psi_{v 1}(t) \sin \frac{\pi x}{L} .
$$

For the midpoint of the curved beam, $x=L / 2$, the vertical displacement is

$$
u_{y}(L / 2, t)=\frac{2 m_{v} g}{m L} \frac{1}{\omega_{v 1}^{2}} \frac{1}{1-S_{v 1}^{2}} \beta \Psi_{v 1}(t) .
$$

The above solution has been obtained by considering only the first mode. More accurate solutions can be obtained through consideration of more modes. In practice, however, the acting time of the vehicles on the curved beam is so short that the moving load problem is by nature a transient problem. As a result, only the first mode will be significantly excited. This is especially true when only the midpoint response of the curved beam is desired, and when the beam is subjected to a series of moving loads, as encountered in high-speed railways. The accuracy of the present solutions will be demonstrated in the numerical example through comparison with the finite element results. 


\subsection{CURVED BEAM SUBJECTED TO A HORIZONTAL MOVING LOAD}

The horizontal moving load $f_{h}$ considered herein is the centrifugal force generated by a vehicle of mass $m_{v}$ moving at speed $v$ along a horizontally curved beam of radius $R$. By taking into account the effect of inertia, the equations of motion for the radial vibration of a curved beam can be modified from equations (1) and (2) as

$$
\begin{aligned}
& m \ddot{u}_{x}+E A\left(u_{x}^{\prime \prime}+\frac{u_{z}^{\prime}}{R}\right)=0, \\
& m \ddot{u}_{2}+E I_{y}\left(u_{z}^{\prime \prime \prime \prime}+2 \frac{u_{z}^{\prime \prime \prime}}{R^{2}}+\frac{u_{z}}{R^{4}}\right)=f_{h} \delta(x-v t),
\end{aligned}
$$

where $f_{h}=m_{v} v^{2} / R$. Similarly, the radial displacement $u_{z}$ can be expressed as the summation of the sine functions that satisfy the boundary conditions

$$
u_{z}(x, t)=\sum_{i=1}^{\infty} q_{z i}(t) \sin \frac{\mathrm{i} \pi x}{L}
$$

in which $q_{z i}$ denotes the $i$ th generalized co-ordinate for the radial displacement. The relation between the radial displacement $u_{z}$ and the axial displacement $u_{x}$ is not arbitrary. By substituting equation (27) into equation (1), the axial displacement $u_{x}$ can be solved as

$$
u_{x}(x, t)=\sum_{i=1}^{\infty}-\frac{q_{z i}(t)}{R}\left(\frac{L}{\mathrm{i} \pi}\right)\left\{1-\cos \frac{\mathrm{i} \pi x}{L}-\left[1-(-1)^{i}\right] \frac{x}{L}\right\} .
$$

If only the first modes are considered, the following may be assumed:

$$
u_{x}(x, t)=q_{x 1}(t)\left(1-\cos \frac{\pi x}{L}-\frac{2 x}{L}\right), \quad u_{z}(x, t)=q_{z 1}(t) \sin \frac{\pi x}{L}
$$

where $q_{x 1}$ and $q_{z 1}$, respectively, denote the first generalized co-ordinate for the axial and radial displacements.

Again, Galerkin's method will be employed to solve the two equations in (26). Namely, by multiplying both sides of equation (26a) by $\delta u_{x}$ and those of equation (26b) by $\delta u_{z}$, making use of the expressions in equations (29), integrating from 0 to $L$, and taking the arbitrary nature of virtual displacements $\delta q_{x 1}$ and $\delta q_{z 1}$, one obtains

$$
\ddot{q}_{x 1}+\bar{a}_{1} q_{x 1}+\bar{a}_{2} q_{z 1}=0, \quad \ddot{q}_{z 1}+\bar{b}_{1} q_{z 1}+\bar{b}_{2} q_{x 1} \delta q_{z 1}=\frac{2 f_{h}}{m L} \sin \frac{\pi v t}{L}
$$

where

$$
\begin{aligned}
& \bar{a}_{1}=\frac{E A \pi^{2}}{L}\left(\frac{4}{\pi^{2}}-\frac{1}{2}\right) / m L\left(\frac{8}{\pi^{2}}-\frac{5}{6}\right), \quad \bar{a}_{2}=\frac{E A \pi}{L}\left(\frac{4}{\pi^{2}}-\frac{1}{2}\right) / m L\left(\frac{8}{\pi^{2}}-\frac{5}{6}\right), \\
& \bar{b}_{1}=\frac{E I_{y}}{m}\left[\left(\frac{\pi}{L}\right)^{2}-\frac{1}{R^{2}}\right]^{2}+\frac{E A}{m R^{2}}, \quad \bar{b}_{2}=\frac{E A}{m R L}\left(\pi-\frac{8}{\pi}\right) .
\end{aligned}
$$


As was stated, the general solutions to equations (30) consist of two parts,

$$
q_{x 1}=q_{x 1 h}+q_{x 1 p}, \quad q_{z 1}=q_{z 1 h}+q_{z 1 p} .
$$

The homogenous parts can be given as

$$
q_{x 1 h}=\bar{h}_{1} \sin \omega_{h 1} t+\bar{h}_{2} \cos \omega_{h 2} t, \quad q_{z 1 h}=\bar{k}_{1} \sin \omega_{h 1} t+\bar{k}_{2} \cos \omega_{h 2} t,
$$

where $\omega_{h 1}$ denotes the frequency of vibration of the beam on the horizontal plane and the coefficients $\bar{h}_{1}, \bar{h}_{2}, \bar{k}_{1}, \bar{k}_{2}$, are to be determined from the initial conditions. Substituting equations (33) into equations (30) and dropping the term containing $f_{h}$ yields

$$
\left[\begin{array}{cc}
\bar{a}_{1}-\omega_{h 1}^{2} & \bar{a}_{2} \\
\bar{b}_{2} & \bar{b}_{1}-\omega_{h 1}^{2}
\end{array}\right]\left\{\begin{array}{l}
q_{x 1 h} \\
q_{z 1 h}
\end{array}\right\}=\left\{\begin{array}{l}
0 \\
0
\end{array}\right\} .
$$

By letting the determinant equal zero, the vibration frequency $\omega_{h 1}$ can be solved as [26]

$$
\omega_{h 1}=\sqrt{\frac{\bar{a}_{1}+\bar{b}_{1}-\sqrt{\left(\bar{a}_{1}-\bar{b}_{1}\right)^{2}+4 \bar{a}_{2} \bar{b}_{2}}}{2} .}
$$

The particular solutions can be given as

$$
q_{x 1 p}=p_{x 1} \sin \frac{\pi v t}{L}, \quad q_{z 1 p}=p_{z 1} \sin \frac{\pi v t}{L}
$$

Substituting the preceding expressions for $q_{x 1 p}$ and $q_{z 1 p}$ into equations (30) yields

$$
\left[\begin{array}{cc}
\bar{a}_{1}-(\pi v / L)^{2} & \bar{a}_{2} \\
\bar{b}_{2} & \bar{b}_{1}-(\pi v / L)^{2}
\end{array}\right]\left\{\begin{array}{c}
p_{x 1} \\
p_{z 1}
\end{array}\right\}=\left\{\begin{array}{c}
0 \\
\frac{2 f_{h}}{m L}
\end{array}\right\}
$$

from which the generalized co-ordinate $p_{z 1}$ can be solved as

$$
p_{z 1}=\frac{2 f_{h}}{m L} \frac{1}{\omega_{h 1}^{2}} \frac{1}{1-S_{h 1}^{2}} a
$$

where

$$
S_{h 1}=\frac{\pi v}{L \omega_{h 1}}, \quad \alpha=\frac{\bar{a}_{1}-(\pi v / L)^{2}}{\bar{a}_{1}+\bar{b}_{1}-\omega_{h 1}^{2}-(\pi v / L)^{2}} .
$$

Here, $S_{h 1}$ denotes the speed parameter for the horizontal vibration of the curved beam, which is defined as the ratio of the driving frequency, $\pi v / L$, to the fundamental frequency $\omega_{h 1}$ of the beam.

By assumption, the zero initial conditions must be obeyed by the general solution $q_{z 1}$, or the one given in equation (32b). From these conditions, the coefficients of the homogeneous solution $q_{z 1 h}$ can be determined as $\bar{k}_{1}=-p_{z 1} S_{h 1}$ and $\bar{k}_{2}=0$. By the relation $f_{h}=m_{v} v^{2} / R$ and using equation (38), the radial displacement $q_{z 1}$ can be derived as

$$
q_{z 1}(t)=\frac{2 m_{v} \varphi}{m \pi^{2}} \frac{S_{h 1}^{2}}{1-S_{h 1}^{2}} \alpha \Psi_{h 1}(t)
$$


where $\varphi=L / R$ for the curved beam and the amplitude function $\Psi_{h 1}(t)$ is

$$
\Psi_{h 1}(t)=\sin \frac{\pi v t}{L}-S_{h 1} \sin \omega_{h 1} t .
$$

As a result, the radial displacement of the curved beam is

$$
u_{z}(x, t)=\frac{2 m_{v} \varphi}{m \pi^{2}} \frac{S_{h 1}^{2}}{1-S_{h 1}^{2}} \alpha \Psi_{h 1}(t) \sin \frac{\pi x}{L} .
$$

Of interest is the fact that when the vehicle speed $v$ approaches zero, $S_{h 1} \rightarrow 0$, the radial displacement $u_{z}(x, t)$ of the curved beam also approaches zero. In contrast, the vertical displacement $u_{y}(x, t)$ as given in equation (24) approaches a constant under the same condition. This can be realized if one notes that as the vehicle speed $v$ reduces to zero, so does the horizontal moving load $f_{h}$, as there is no centrifugal force. However, the vertical moving load $f_{v}$ remains constant, regardless of the vehicle speed.

\section{UNIFIED EXPRESSIONS FOR VERTICAL AND RADIAL VIBRATIONS}

The solutions for the vertical and radial displacements, $u_{y}(x, t)$ and $u_{z}(x, t)$, in equations (24) and (42) are similar in form. During the travel time $L / v$ of the vehicle on the beam, the two equations can be collectively expressed as

$$
U(x, t)=P \Psi(t) H(t) \sin \frac{\pi x}{L} \text { for } 0 \leqslant t \leqslant L / v,
$$

where $H(t)$ is a unit step function, indicating that the function $\Psi(t)$ is on at $t=0$. For the vertical vibration case, $U(x, t)$ should be interpreted as $u_{y}(x, t), P$ as $-\left(2 m_{v} g / m L\right)\left(1 / \omega_{v 1}^{2}\right)$ $\left[1 /\left(1-S_{v 1}^{2}\right)\right] \beta$, and $\Psi(t)$ as $\Psi_{v 1}(t)$. For the horizontal vibration case, $U(x, t)$ should be interpreted as $u_{z}(x, t), P$ as $\left(2 m_{v} \varphi / m L\right)\left[S_{h 1}^{2} /\left(1-S_{h 1}^{2}\right)\right] \alpha$, and $\Psi(t)$ as $\Psi_{h 1}(t)$. For $t>L / v$, the beam reaches a state of free vibration, as the moving load already leaves the beam. By the fact that $L / v$ denotes half of the period of the moving load over the beam, the response of the beam at this stage is

$$
U(x, t)=P\left[\Psi(t) H(t)+\Psi\left(t-\frac{L}{v}\right) H\left(t-\frac{L}{v}\right)\right] \sin \frac{\pi x}{L} \quad \text { for } t>\frac{L}{v} .
$$

Here, the amplitude function $\Psi(t)$ is

$$
\Psi(t)=\sin \frac{\pi v t}{L}-S_{1} \sin \omega_{1} t
$$

where the frequency $\omega_{1}$ and speed parameter $S_{1}$ should be interpreted as $\omega_{v 1}$ and $S_{v 1}$ for the vertical vibration, and as $\omega_{h 1}$ and $S_{h 1}$ for the horizontal vibration. By substitution of equation (45) for $\Psi(t)$, noting that $H(t)=H(t-L / v)$ for $t>L / v, \sin (\pi v t / L)+$ $\sin [\pi v(t-L / v) / L]=0 \quad$ and $\quad \sin a+\sin b=2 \sin [(a+b) / 2] \cos [(a-b) / 2]$, one can rearrange equation (44) as

$$
U(x, t)=-2 P S_{1} \cos \frac{\omega_{1} L}{2 v} \sin \omega_{1}\left(t-\frac{L}{2 v}\right) H\left(t-\frac{L}{2 v}\right) \sin \frac{\pi v t}{L} \quad \text { for } t>\frac{L}{v}
$$


This is exactly the residual response of the beam after the moving load has left the beam, based on the first-mode approximation.

By letting $\cos \left(\omega_{1} L / 2 v\right)=0$ or $\omega_{1} L / 2 v=(2 i-1) \pi / 2$, with $i=1,2,3, \ldots$, one observes that the residual response of the beam reduces to zero. Such a condition is equivalent to

$$
S_{1}=\frac{1}{2 i-1} \text { for } i=1,2,3, \ldots .
$$

Theoretically speaking, if the condition (47) is met, the residual response of the beam simply vanishes. For this reason, the condition (47) is referred to as the condition of cancellation.

\section{SOLUTIONS FOR MULTI MOVING LOADS}

Based on the assumption of small deformations, the vibration of a beam caused by a series of moving loads can be obtained as the superposition of the vibration induced by each of the moving loads. In such a process, care must be taken regarding the acting period of each moving load on the beam and the time lag between any two consecutive loads.

Consider $N$ identical masses of interval $d$ moving at speed $v$. As shown in Figure 3, each of the masses will induce a vertical load $f_{v}$ and a centrifugal force $f_{h}$. Assuming that the first moving load enters the beam at $t=0$, the time lag for the $j$ th moving load is $t_{j}=(j-1) d / v$. Thus, the residual displacement of the midpoint of the beam caused by the $j$ th moving load is

$$
U_{j}(L / 2, t)=P\left[\Psi\left(t-t_{j}\right) H\left(t-t_{j}\right)+\Psi\left(t-t_{j}-L / v\right) H\left(t-t_{j}-L / v\right)\right] .
$$

The most critical condition for the beam occurs when the first $N-1$ masses have left and only the $N$ th mass is acting on the beam. The time interval for such a case is $\max \left(t_{N}, t_{N-1}+L / v\right)<t<L / v$, as shown in Figure 4. For this case, the midpoint response of the beam is composed of two parts. The first part relates to the forced vibration caused by the $N$ th moving load, which can be obtained by letting $x=L / 2$ and replacing $t$ by $t-t_{N}$ in equation (43), i.e.,

$$
U_{1}(L / 2, t)=P \Psi\left(t-t_{N}\right) H\left(t-t_{N}\right) \text { for } 0<t-t_{N}<L / v .
$$

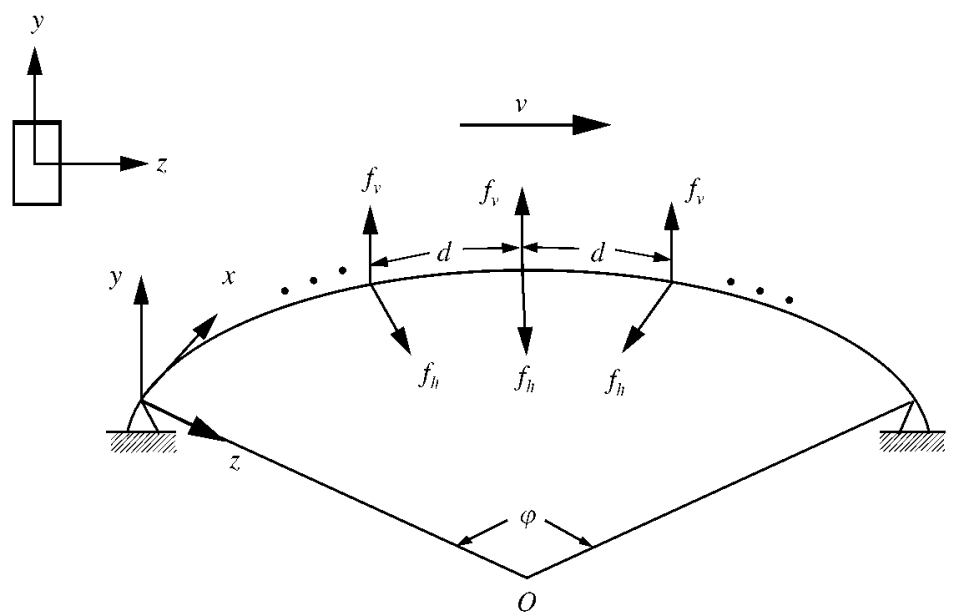

Figure 3. Curved beam subjected to equi-spaced moving loads. 


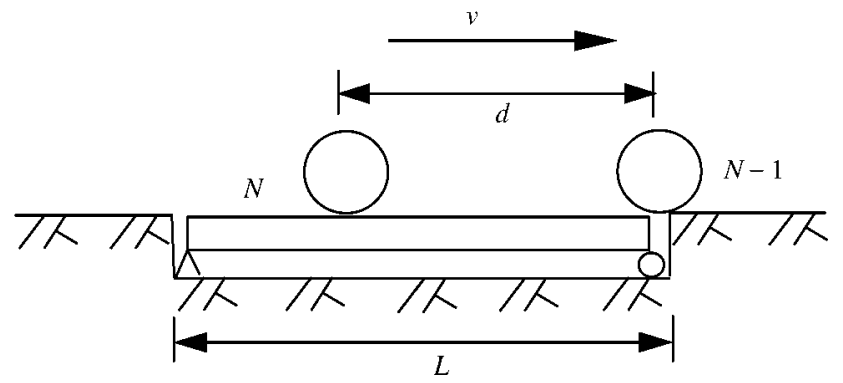

Figure 4. Critical loading case.

The second part is simply the residual vibration caused by the $N-1$ moving loads that passed the beam. This can be obtained by letting $x=L / 2$ and replacing $t$ by $t-t_{j}$ in equation (46), and then summing the responses induced by the moving loads from $j=1$ to $N-1$. Note that when $H\left(t-t_{N-1}-L / v\right)=1$, it can be ascertained that $H\left(t-t_{j}-L / v\right)=$ 1 for $j=1,2, \ldots, N-1$. Thus, $H\left(t-t_{j}-L / v\right)$ for $j=1,2, \ldots, N-1$ can be replaced by $H\left(t-t_{N-1}-L / v\right)$. The following is the result for part two:

$$
\begin{aligned}
U_{2}\left(\frac{L}{2}, t\right)= & \sum_{j=1}^{N-1}\left\{-2 P S_{1} \cos \frac{\omega_{1} L}{2 v} \sin \omega_{1}\left[\left(t-t_{j}\right)-\frac{L}{2 v}\right]\right\} H\left(t-t_{N-1}-\frac{L}{v}\right) \\
& \text { for } t-t_{N-1} \geqslant L / v
\end{aligned}
$$

which can be manipulated to remove the sign of summation as [27]

$$
\begin{aligned}
U_{2}\left(\frac{L}{2}, t\right)= & -2 P S_{1} \cos \frac{\omega_{1} L}{2 v}\left[\sin \omega_{1}\left(t-\frac{L}{2 v}\right)+\sin \omega_{1}\left(\frac{N-2}{2} \frac{d}{v}\right)\right. \\
& \left.\times \sin \omega_{1}\left(t-\frac{L}{2 v}-\frac{N-1}{2} \frac{d}{v}\right) \sin ^{-1} \frac{\omega_{1} d}{2 v}\right] H\left(t-t_{N-1}-\frac{L}{v}\right) \\
& \text { for } t-t_{N-1}>L / v .
\end{aligned}
$$

Consequently, the midpoint response of the beam under the action of the $N$ th moving load can be computed as the summation of $U_{1}$ and $U_{2}$ given in equations (49) and (51), respectively.

\section{CONDITIONS OF RESONANCE AND CANCELLATION}

The phenomena of resonance and cancellation relate to the free vibration response $U_{2}$ caused by the $N-1$ moving loads that have passed the beam. From equation (51), one observes that the response of the beam reaches a maximum when the denominator of some terms vanishes, i.e., when $\sin \left(\omega_{1} d / 2 v\right)=0$ or when $\omega_{1} d / 2 v=i \pi$, with $i=1,2,3, \ldots$, or equivalently,

$$
S_{1}=\frac{1}{2 i} \frac{d}{L} \quad \text { for } i=1,2,3, \ldots
$$


This is exactly the condition for resonance of the beam to occur. For the case with $\sin \left(\omega_{1} d / 2 v\right)=0$, expression (51) becomes indeterminate. By the condition $\sin \left(\omega_{1} d / 2 v\right)=0$ and L 'Hospital's rule, equation (51) can be manipulated to yield

$$
U_{2}\left(\frac{L}{2}, t\right)=-2 P(N-1) \cos \frac{\omega_{1} L}{2 v} \sin \omega_{1}\left(t-\frac{L}{2 v}\right) H\left(t-t_{N-1}-\frac{L}{v}\right) .
$$

Here, it is interesting to note that the midpoint response of the beam increases as there are more moving loads passing the beam.

On the other hand, one observes from equation (51) that whenever $\cos \left(\omega_{1} L / 2 v\right)=0$, or $\omega_{1} L / 2 v=(2 i-1) \pi / 2$, with $i=1,2,3, \ldots$, or equivalently the one given in equation (47), the free vibration response $U_{2}(L / 2, t)$ reduces to zero, which means that no residual response will be generated by the moving loads having passed the beam. Such a condition has been referred to as the condition of cancellation. The conditions of resonance and cancellation, as given in equations (52) and (47), are identical in form for both the vertical and horizontal oscillations in terms of the speed parameter $S_{1}$. However, because the vertical and horizontal vibration frequencies, $\omega_{v 1}$ and $\omega_{h 1}$, are generally different for the beam, the resonance or cancellation phenomena for the two directions do not occur at the same speed $v$.

\section{NUMERICAL EXAMPLES}

The curved beam considered herein is simply supported, of length $L=24 \mathrm{~m}$ and subtended angle $\varphi=30^{\circ}$. The following properties are assumed for the cross-section: $A=9 \mathrm{~m}^{2}, \quad I_{y}=18.75 \mathrm{~m}^{4}, I_{z}=2.43 \mathrm{~m}^{4}, J=21.18 \mathrm{~m}^{4}, E=32.2 \mathrm{GPa}, v$ (the Poisson ratio) $=0.2$, and $\rho=2.4 \mathrm{t} / \mathrm{m}^{3}$. Each vehicle has a mass of $m_{v}=29.9 \mathrm{t}$ and the distance between two adjacent vehicles is $d=25 \mathrm{~m}$. Unless noted otherwise, all the data assumed here will be used throughout this section. For comparison, finite element solutions obtained by approximating the curved beam by 10 piecewise straight beam elements will also be presented. As the straight beam element was derived from the straight beam theory, the results obtained from the finite element analysis are totally independent of the present analytical results based on the curved beam theory, as represented by equation (1)-(4).

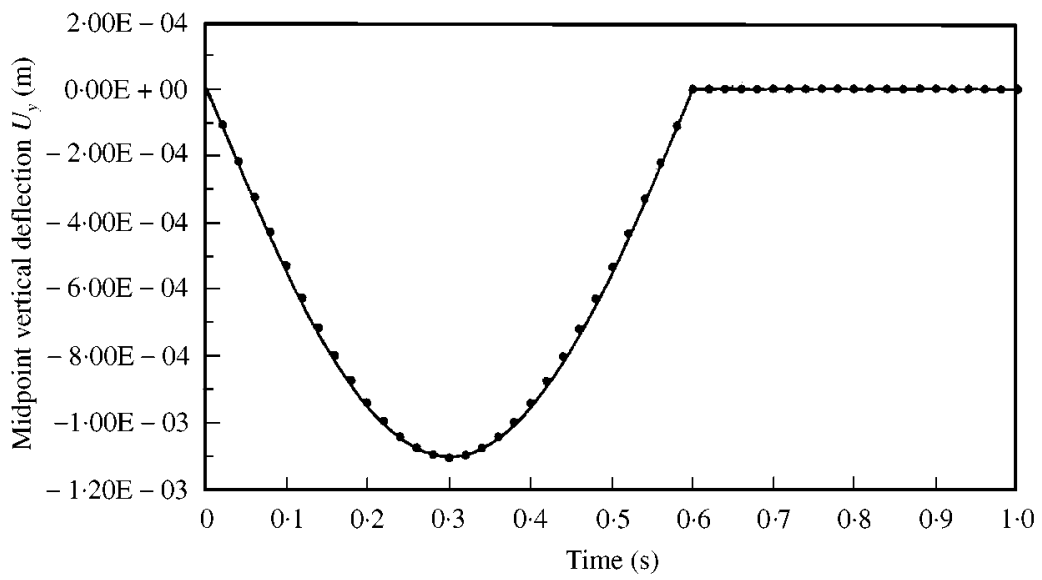

Figure 5. Midpoint static vertical response of curved beam (one moving mass): - , analytical; $\bullet$, FEM. 


\subsection{COMPARISON OF ANALYTIC WITH FINITE ELEMENT SOLUTIONS}

Consider a single moving mass with speed $v=40 \mathrm{~m} / \mathrm{s}$, which will generate a gravitational force and a centrifugal force. The frequencies of vibration computed for the two directions of the beam using equations (16) and (35) are $\omega_{v 1}=32.10 \mathrm{rad} / \mathrm{s}$ and $\omega_{h 1}=115.4 \mathrm{rad} / \mathrm{s}$, compared with the finite element results of $\omega_{v 1}=32.24 \mathrm{rad} / \mathrm{s}$ and $\omega_{h 1}=116.61 \mathrm{rad} / \mathrm{s}$.

The static vertical response, dynamic vertical response and dynamic horizontal response of the midpoint of the curved beam have been plotted in Figures 5-7, along with the finite element solutions. As can be seen, the present solutions agree very well with the numerical ones, which is a demonstration of the accuracy of the present solutions considering only the first mode. Noting that the acting time of the mass is $L / v=0.6 \mathrm{~s}$, one can easily distinguish between the forced vibration of the beam and the residual response in Figures 5-7; the latter does not decay since the damping effect was ignored. On the other hand, it is observed that the vertical response is much larger than that of the horizontal one, indicating that the vertical response can be more easily excited by the load moving at $v=40 \mathrm{~m} / \mathrm{s}$.

\subsection{PHENOMENON OF CANCELLATION UNDER SINGLE OR MULTI MOVING MASSES}

As an illustration, we shall let $i=2$ in equation (47) and compute the speed of cancellation as $S_{1}=0.333$. By the definition for $S_{1}$ in equations (20a) and (39a), along with $\omega_{v 1}=32.24 \mathrm{rad} / \mathrm{s}$ and $\omega_{h 1}=116.61 \mathrm{rad} / \mathrm{s}$, the speed of cancellation can be computed for the two directions as $v_{v}=82 \mathrm{~m} / \mathrm{s}$ and $v_{h}=297 \mathrm{~m} / \mathrm{s}$. Considering a single mass moving at these speeds, each for one direction, the time-history midpoint response computed for the two directions is plotted in Figures 8 and 9. As can be seen, the residual response induced by the moving mass after it leaves the beam, i.e., after 0.29 and $0.08 \mathrm{~s}$, respectively, for the vertical and horizontal directions, deviates slightly from the theoretical value of zero, due to neglect of the higher modes. The close agreement of the present solutions with the finite element ones indicates that the effect of higher modes is generally small.

Consider next the case of eight moving masses and use the same speeds of cancellation, i.e., $v_{v}=82 \mathrm{~m} / \mathrm{s}$ and $v_{h}=297 \mathrm{~m} / \mathrm{s}$, for the two directions. The time-history response computed for the midpoint response for the two directions has been plotted in Figures 10

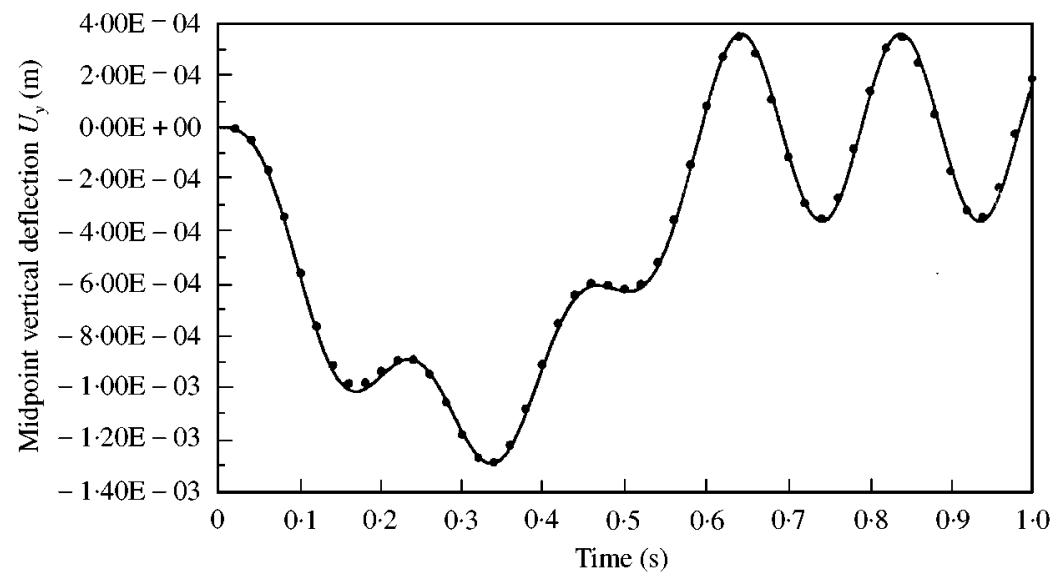

Figure 6. Midpoint dynamic vertical response of curved beam (one moving mass): ——, analytical; •, FEM. 


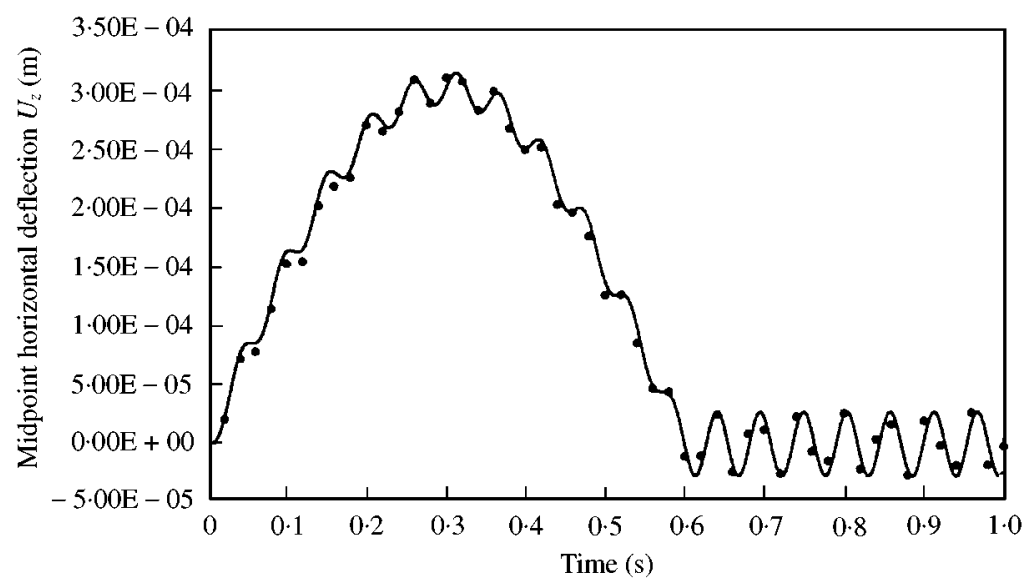

Figure 7. Midpoint dynamic horizontal response of curved beam (one moving mass): — , analytical; $\bullet$, FEM.

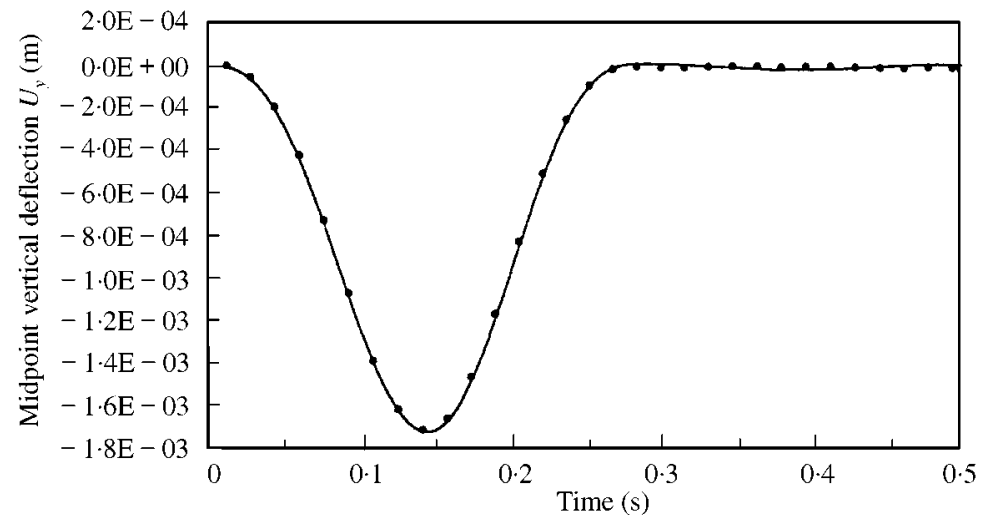

Figure 8. Phenomenon of cancellation for vertical direction (one moving mass): — , analytical; $\bullet$, FEM.

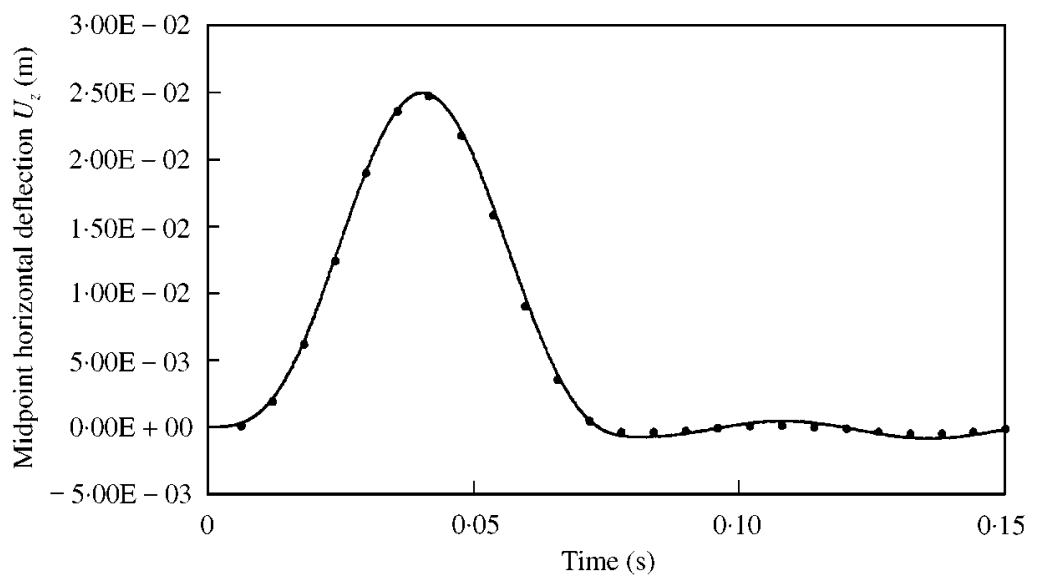

Figure 9. Phenomenon of cancellation for horizontal direction (one moving mass): - , analytical; $\bullet$, FEM. 


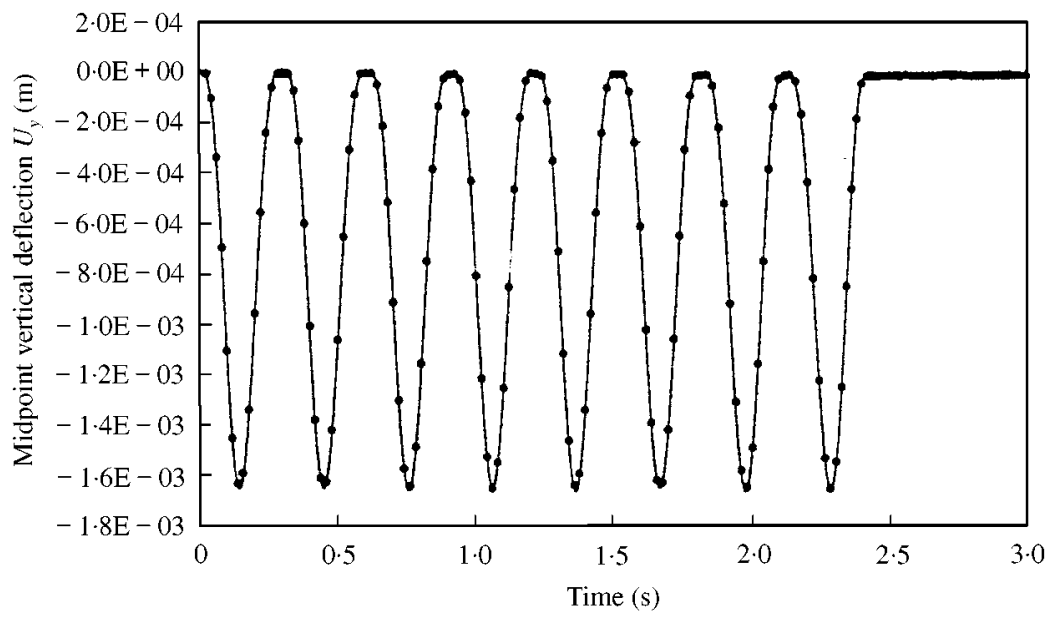

Figure 10. Phenomenon of cancellation for vertical direction (eight moving masses): ——, analytical; •, FEM.

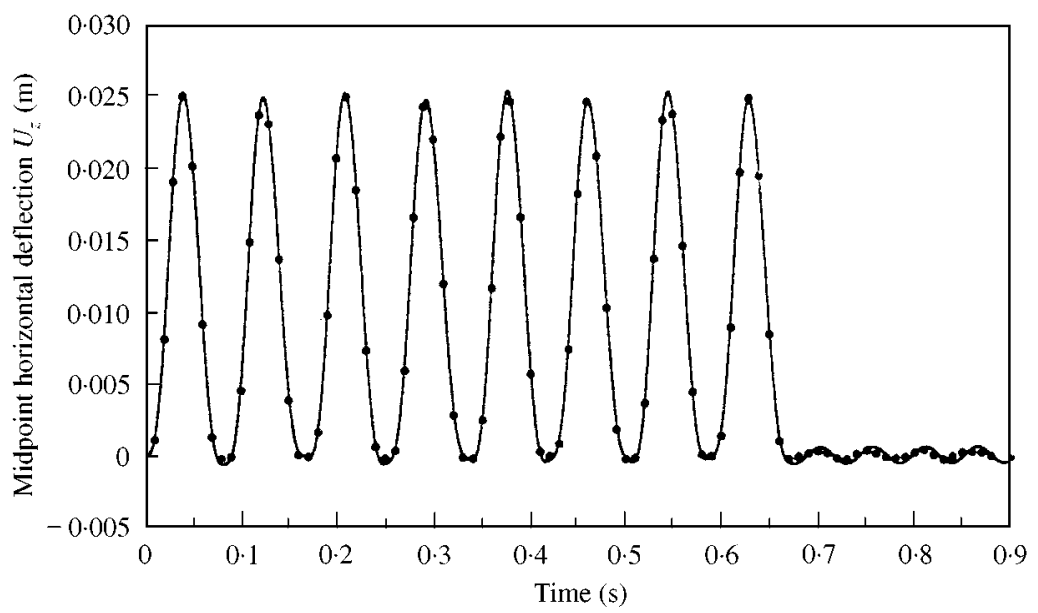

Figure 11. Phenomenon of cancellation for horizontal direction (eight moving masses): —_, analytical; •, FEM.

and 11. The time durations for all the moving loads to depart from the beam are 2.43 and $0.67 \mathrm{~s}$ for the vertical and horizontal directions, respectively. In the two figures, it is observed that there is a total of eight peaks, each of which corresponds to one moving load. Moreover, the residual responses for both directions remain negligibly small, as was expected. Finally, the effect of higher modes appear to be generally small, as the present solutions agree very well with the finite element ones.

\subsection{PHENOMENON OF RESONANCE UNDER MULTI MOVING MASSES}

Consider also the case of eight moving masses. Using $d=25 \mathrm{~m}$ and $L=24 \mathrm{~m}$, the resonance speed computed from equation (52) for $i=1$ is $S_{1}=0.521$. By the use of equations (20a) and (39a), along with $\omega_{v 1}=32 \cdot 24 \mathrm{rad} / \mathrm{s}$ and $\omega_{h 1}=116.61 \mathrm{rad} / \mathrm{s}$, the corresponding speeds computed of the moving masses for two directions are $v_{v}=128 \mathrm{~m} / \mathrm{s}$ 
and $v_{h}=464 \mathrm{~m} / \mathrm{s}$. In Figures 12 and 13, the time-history response obtained for the vehicles travelling at the resonance speed for each direction has been plotted. The fact that the response increases for the two directions as there are more masses passing the beam is typical of the resonance phenomenon. After all the masses pass the beam, i.e., after 1.55 and $0.43 \mathrm{~s}$ for the vertical and horizontal directions, respectively, the beam tends to oscillate with the largest amplitude that has been excited, as no damping is assumed. Again, the present solutions match very well the finite element solutions.

\subsection{I-S PLOT-IMPACT EFFECT CAUSED BY MOVING LOADS}

By letting $R_{d}(x)$ and $R_{s}(x)$, respectively, denote the maximum dynamic and static deflection of the beam at position $x$ due to the action of the moving loads, the impact factor $I$ for the deflection of a beam subjected to the moving loads can be defined as

$$
I=\frac{R_{d}(x)-R_{s}(x)}{R_{s}(x)} .
$$

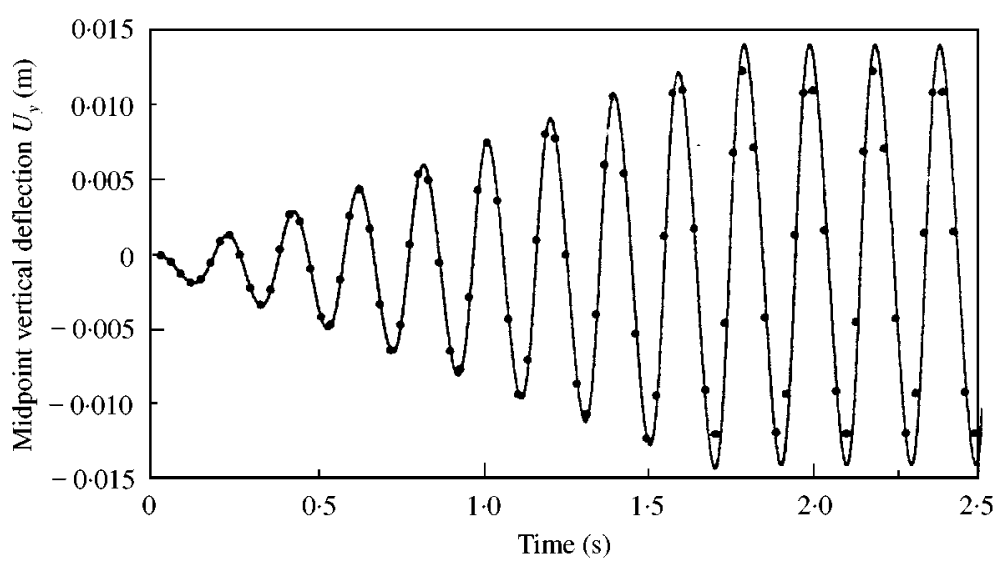

Figure 12. Phenomenon of resonance for vertical direction (eight moving masses): —

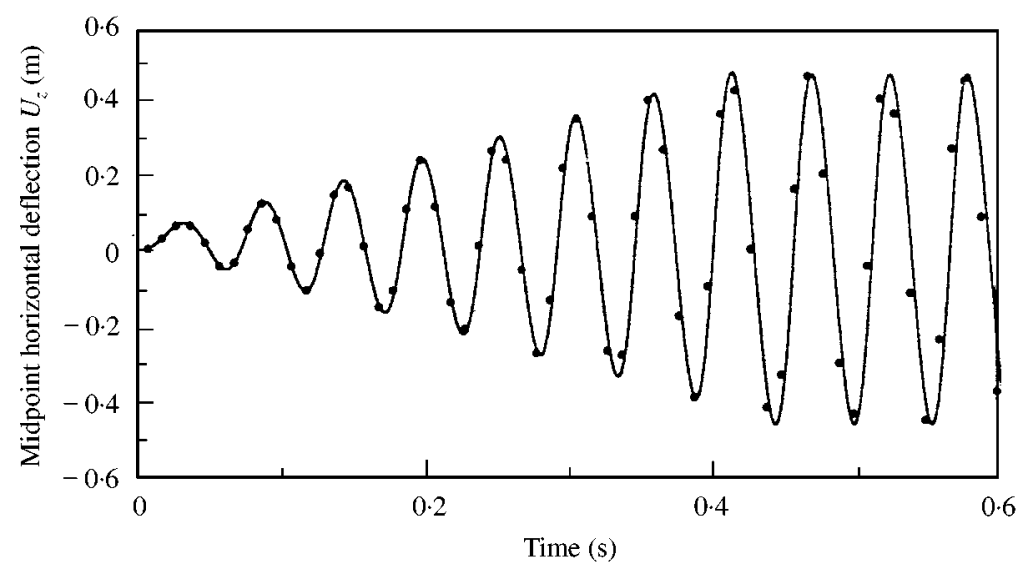

Figure 13. Phenomenon of resonance for horizontal direction (eight moving masses): — , analytical; $\bullet$, FEM. 
For simple beams, both the maximum static and dynamic deflections will occur at the midpoint. Consider the case of eight equally spaced moving masses. The impact response of the vertical deflection of the beam versus the speed parameter of the moving masses has been plotted in Figure 14, along with the finite element solutions. The impact response of the horizontal deflection can hardly be plotted, because of the lack of a static centrifugal force. However, if the centrifugal force $f_{h}$ computed as $m_{v} v^{2} / R$ can be treated as if it were a static force, the impact response of the horizontal deflection can be computed as well (not shown), which appears to be quite similar to that presented in Figure 14 for the vertical vibration [26]. In Figure 14, it is confirmed that the present solutions agree very well with the finite element results. By substituting $d=25 \mathrm{~m}$ and $L=24 \mathrm{~m}$ into equation (52), the resonance speeds computed for the two directions are $S_{1}=0 \cdot 521,0 \cdot 260,0 \cdot 174,0 \cdot 130, \ldots$, which are close to the points where the peaks occur in Figure 14. On the other hand, from equation (47) the speeds of cancellation can be computed as $S_{1}=1 \cdot 000,0 \cdot 333,0 \cdot 2000$, $0 \cdot 143, \ldots$, which are also close to the points where the minimum values occur in Figure 14.

As was noted, the impact response for the horizontal vibration of the curved beam is unreal, since there is no static centrifugal force. In Figure 15, the maximum horizontal deflection for the midpoint of the curved beam has been plotted. As can be seen, the speeds

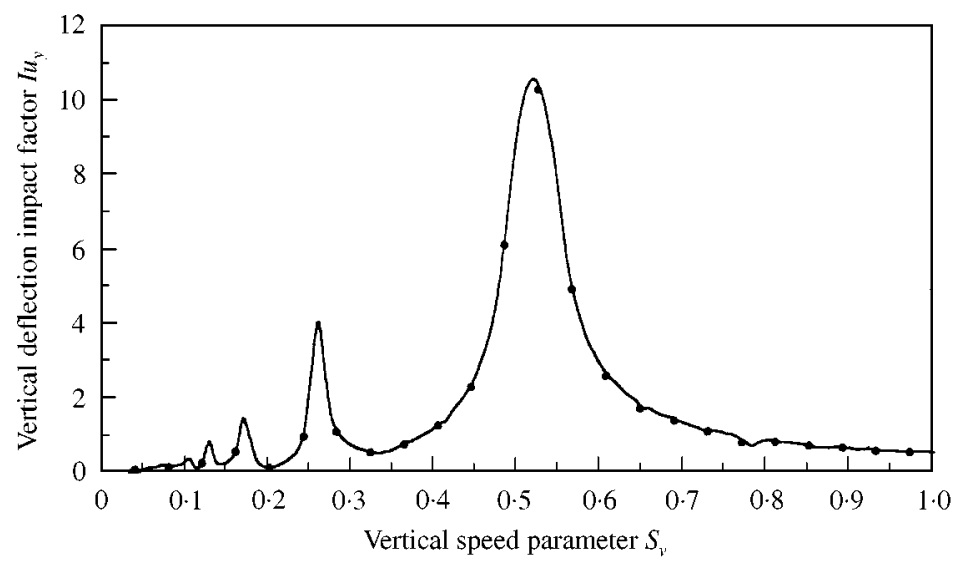

Figure 14. Impact factor for midpoint vertical deflection (eight moving masses): — , analytical; $\bullet$, FEM.

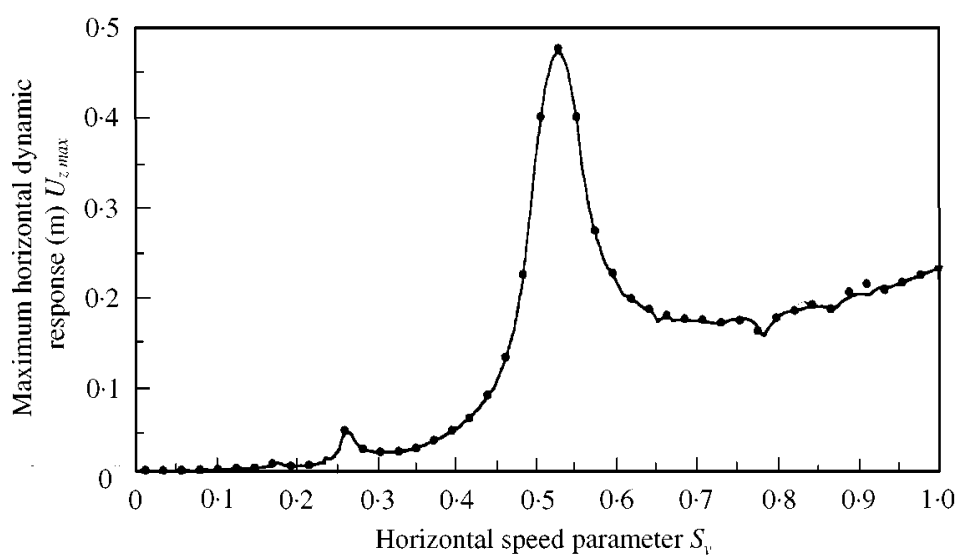

Figure 15. Maximum response for midpoint horizontal deflection (eight moving masses): — 
for the maximum and minimum values to occur are generally consistent with those of Figure 14, although they are much less clear.

\section{CONCLUDING REMARKS}

In this paper, a general theory has been presented for treating the vibration of a horizontally curved beam subjected to moving masses, each of which is simulated as a gravitational force and a centrifugal force. The problem has been solved in an analytical but approximate manner considering the contribution of the first mode of vibration. The accuracy of the present approach has been confirmed by an independent finite element analysis that, by nature, considers all modes of vibration. The advantage of the present approach is that it provides clear physical insights into the various vibration phenomena induced by vehicles, in particular, the phenomena of resonance and cancellation, and allows us to identify the key parameters involved. The solution established herein for the horizontal vibration of curved beams subjected to the centrifugal force is believed to be new in the literature.

\section{ACKNOWLEDGMENTS}

The research reported herein was sponsored by the National Science Council through Grant No. NSC87-2211-E002-065.

\section{REFERENCES}

1. R. WILlis 1849 Appendix to the Report of the Commissioners Appointed to Inquire into the Application of Iron to Railway Structures. London, UK: H.M. Stationary Office.

2. G. G. STOKES 1849 Transaction of the Cambridge Philosophical Society 8, 707-735. Discussion of a differential equation relating to the breaking of railway bridges.

3. S. P. Timoshenko 1922 Philosophical Magazine, Series 6, 43, 1018-1019. On the forced vibration of bridges.

4. H. H. JefFCotT 1929 Philosophical Magazine, Series 7, 8, 66-97. On the vibrations of beams under the action of moving loads.

5. M. M. StAnišIĆ and J. C. HARdin 1969 Journal of Franklin Institute 287, 115-123. On the response of beams to an arbitrary number of concentrated moving masses.

6. E. C. Ting, J. Genin and J. H. Ginsberg 1974 Journal of Sound and Vibration 33, 49-58. A general algorithm for the moving mass problem.

7. C. P. TAN and S. SHORE 1986 Journal of Structural Division, American Society of Civil Engineers 94, 2135-2151. Response of horizontally curved bridge to moving load.

8. A. S. Veletsos and T. HuAng 1970 Journal of Engineering Mechanics Division, American Society of Civil Engineers 96, 593-620. Analysis of dynamic response of highway bridges.

9. T. E. Blejwas, C. C. Feng and R. S. Ayre 1979 Journal of Sound and Vibration 67, 513-521. Dynamic interaction of moving vehicles and structures.

10. L. FRÝBA 1972 Vibration of Solids and Structures under Moving Loads. Groningen, Netherlands: Noordhoff International Publishing.

11. K. H. ChU, V. K. GARG and T. L. WANG 1986 Journal of Structural Engineering, American Society of Civil Engineers 112, 1036-1051. Impact in railway prestressed concrete bridges.

12. E. S. Hwang and A. S. NowaK 1991 Journal of Structural Engineering, American Society of Civil Engineers 117,1413-1434. Simulation of dynamic load for bridges.

13. N. H. Galdos, D. R. Schelling and M. A. SAhin 1993 Journal of Structural Engineering, American Society of Civil Engineers 119, 1917-1934. Methodology for impact factor of horizontally curved box bridges.

14. P. K. Chatterjee, T. K. DatTa and C. S. Surana 1994 Journal of Structural Engineering, American Society of Civil Engineers 120, 681-703. Vibration of suspension bridges under vehicular movement. 
15. M. J. Inbanathan and M. Wieland 1987 Journal of Structural Engineering, American Society of Civil Engineers 113, 1994-2008. Bridge vibrations due to vehicle moving over rough surface.

16. Y. B. YANG and B. H. LIN 1995 Journal of Structural Engineering, American Society of Civil Engineers 121, 1636-1643. Vehicle-bridge interaction analysis by dynamic condensation method.

17. Y. B. YANG and J. D. YAU 1997 Journal of Structural Engineering, American Society of Civil Engineers 123, 1512-1518. Vehicle-bridge interaction element for dynamic analysis (errata: 124, 479).

18. G. Diana and F. Cheli 1989 Vehicle System Dynamics 18, 71-106. Dynamic interaction of railway systems with large bridges.

19. P. Paultre, O. Chatllal and J. Proulx 1992 Canadian Journal of Civil Engineering 19, 260-278. Bridge dynamics and dynamic amplification factors - a review of analytical and experimental findings.

20. Y. B. YANG and J. D. YAU 1998 Proceedings of the National Science Council, ROC-Part A: Physical Sciences and Engineering 22, 214-224. Vehicle-bridge interactions and applications to high speed rail bridges.

21. L. FRÝBA 1996 Dynamics of Railway Bridges. London: Thomas Telford.

22. C. P. TAN and S. SHORE 1968 Journal of Structural Division, American Society of Civil Engineers $\mathbf{9 4 ,}$ 761-781. Dynamic response of a horizontally curved bridges.

23. J. Genin, E. C. Ting and Z. VAFA 1982 Journal of Sound and Vibration 65, 569-575. Curved bridge response due to a moving vehicle.

24. Y. B. YANG and S. R. KuO 1987 Journal of Structural Engineering, American Society of Civil Engineers 113, 1185-1202. Effect of curvature on stability of curved beams.

25. Y. B. YANG and S. R. KUO 1994 Theory and Analysis of Nonlinear Framed Structures. Englewood Cliffs, NJ: Prentice-Hall, Chapter 7.

26. C. M. Wu 1998. Master's Thesis, Department of Civil Engineering, National Taiwan University, Taipei, Taiwan, $R O C$, Dynamic response of curved bridges due to high speed trains and vibration of continuous bridges (in Chinese).

27. Y. B. YANG, J. D. YAU and L. C. HSU 1997 Vibration of simple beams due to trains moving at high speeds. Engineering Structures 19, 936-944. 